\title{
“I AM NOT FULLY MEDICALIZED.”: A QUALITATIVE STUDY OF POST-NATAL CARE AMONG MALAYSIAN CHILD-BIRTHING WOMEN IN THE UNITED KINGDOM
}

\author{
Fitrianti $\mathrm{Y}^{*}$
}

National Institute of Health Research and Development, Indonesia

\begin{abstract}
Obstetric medicine and reproductive technology have been spread out worldwide and become the symbol of modernization. Its expansion might displace the traditional treatments which mostly are practiced by the people in developing countries. However, the Malaysian women who lived in a Western country and had a well-educated background still practiced the traditional treatments after giving birth. The study was conducted in 2016 at Durham, a county in the United Kingdom, and it utilized qualitative research by interviewing five Malaysian women who had a birth experience in the United Kingdom. The result of the study revealed that heating the body with hot stone has still mostly practiced by Malaysian women even living in the United Kingdom, where there were optional sophisticated technology and qualified medical professional. In addition, some of them still obeyed the recommended and prohibited foods ruled by the origin culture during the postpartum period. The treatment was conducted at home supported by the family and colleagues whose the same ethnicity and nationality. In conclusion, the national boundaries, high education, and the existence of sophisticated health technology and qualified medical professional are irrelated to why people still undertake traditional treatments. The treatment was primarily chosen because of its health effects on the body after treatments. Therefore, health policymakers have to know and consider the migrant's cultural values in order to make the health system convenient and appropriate to either the migrants' health. In addition, the study needs further research to find the effectiveness and efficacy of traditional treatments to women's health.
\end{abstract}

Keywords: traditional treatments, postpartum period, humoral system, heat therapy, cultural value, Malaysian culture

\section{Introduction}

In the twentieth century, obstetric medicine and reproductive technology increased rapidly, aligning with the rapid rise of science and industry in general (Henley-Einion, 2003). Dundes (2003) indicated that the biomedical model of birth, which has become a dominant practice in the US and Western countries, has been exported to developing countries, moreover in the globalization era. Globalization generates medicalization, where medicalization, as Illich (Henley-Einion, 2003: 174) pointed out, is linked to industrialization, which has produced sophisticated technologies, as a symbol of modernization. The rise of medical knowledge and technology impacts society across the world either in developed or developing countries. Through globalization - as Browner and Sargent (2011) argue that the term globalization is used to describe the movement or flow of information, products, 
commodities, capital, and people across multinational boundaries - medical knowledge and technologies, in particular for childbirth, are used in every country worldwide.

The expansion of medicalization has been a threat to traditional treatments mostly practiced in developing countries. Modernization, globalization, and migration, which happened worldwide today, might make the people unable to undertake the traditional rituals, moreover, they no longer believe in the traditions (Dennis et al., 2007). From medical perspectives, traditional treatments have been questioned and considered unscientific treatments. However, traditional healing is inevitably practiced by the people in most parts of developing countries, and even though they live in developed countries. Green et al., (2006) who studied the use of traditional Chinese medicine in the United Kingdom revealed that Chinese people in the United Kingdom still looked for traditional Chinese medicines, and even they believed that traditional Chinese healers and medicines were better than Western medicines.

Similarly, the Malaysian women, who lived in the United Kingdom, also looked for the traditional treatments appropriate to their cultures and beliefs. This article will describe the perspective of Malaysian women, on their birth experiences in the United Kingdom. Even though the women lived in a modern country, they still practiced traditional treatments and beliefs. In many cultures, the postpartum period is looked at as a precarious time, because the mother is being vulnerable to have been ill and sick, as a result, the traditional practice should be undertaken by the mother to recover mother's health and avoid illness in later years (Dennis et al., 2007). As Jordan (1992) and Lupton (2007) also argued that every group of people has different ways related to how they perceive and deal with pregnancy and birth.

\section{Research Methods}

This paper is based upon four months of qualitative research conducted in April - July 2016 on Malaysian women living in Durham, a county in the United Kingdom. The postpartum practice described in this paper was further confirmed by the five Malaysian women, who experienced childbirthing in the United Kingdom. An in-depth interview is employed in this study to get the point of women's view regarding their experiences of child-birthing, in particular during the postpartum period in the United Kingdom.

The five Malaysian women involved in this study were chosen by purposive sampling (see table 1), as Bernard (2006) specified in purposive sampling, the researcher decides the aims that $s / h e$ wants the informants to serve. The eligible participants in this research were the women from Malaysia living in the United Kingdom and also had the experience of having a child in the United Kingdom in the last five years (2012-2016). The three of five women were the student of Ph. D. and the other two women accompanied their husbands studied in the United Kingdom, however, these two women were scholars who had accomplished their Bachelor degree in their countries. The five women were asked about their experiences during pregnancy, birthing, and the postpartum period. However, this paper specified only their experiences during postpartum; how they perceived the postpartum period, and considered the practice of their cultural beliefs. 
Table 1: The characteristic of subjects

\begin{tabular}{|l|l|l|l|l|l|l|}
\hline & $\begin{array}{l}\text { Pseud } \\
\text { onym }\end{array}$ & Age & Last Education & Occupation & $\begin{array}{l}\text { Child Birth } \\
\text { Places } \\
\text { (Country) }\end{array}$ & $\begin{array}{l}\text { Normal/ } \\
\text { Caesarean Section }\end{array}$ \\
\hline 1 & Yy & 39 & PhD & $\begin{array}{l}\text { Government } \\
\text { Employee }\end{array}$ & Malaysia and UK & Normal \\
\hline 2 & $\mathrm{Mr}$ & 35 & Bachelor's degree & Housewife & Malaysia and UK & Normal \\
\hline 3 & An & 26 & PhD & Lecturer & UK & Normal with Forceps \\
\hline 4 & As & 26 & Bachelor's degree & Nurse & UK & Miscarriage \\
\hline 5 & Az & 39 & PhD & Lecturer & Malaysia and UK & Normal \\
\hline
\end{tabular}

\section{Results}

\section{Heating Woman's Body}

The five Malaysian women in the United Kingdom, especially those whom I interviewed, gave birth in the hospital assisted by a midwife or medical doctor. After few days of staying in the hospital, the woman went back home and had been visited by a health visitor, who looked at the new-born's developmental weight, asked about the mother's health and feeling, and suggested the mother eat and live healthily. Although the women have been assisted by health professionals and visited by a health visitor, they still confidently practiced the traditional treatments in their homes such as heating their bodies through hot stone and consuming herbal and traditional medicines. The women believed that these traditional treatments and medicines benefited their bodies and health.

The traditional treatment that was still practiced by Malaysian women is heating their bodies with tungku. Tungku is a spherical stone that was brought from Malaysian (see figure 1) and used alternately among Malaysian women who just had given birth. The tungku was heated on a stove or heater then wrapped in leaves, such as daun sirih (betel leaves), and subsequently wrapped in a cotton cloth. After being wrapped, the stone was applied to the post-parturient woman's body and used to massage her abdomen, hands, and feet. Women also asked their husbands to massage their backs with a tungku. The tungku was routinely applied every morning for approximately forty days.

After massaging the body using a tungku, women wrapped their abdomen with a long cloth called barut. Additionally, the inside of the barut, comprised a poultice of turmeric and salt, in order to assist a woman to maintain her health after giving birth. The woman would use the barut all day and subsequently used a fresh barut the following day. When the barut was opened the next day, the woman applied tungku as before, and then once again used barut. The tradition would be employed during the post-partum period, for roughly forty days. The aim of using tungku and barut is to heat a mother's cold body and moreover, to release clotted blood from her body. Women perceived that using tungku and barut was exceedingly advantageous for their health and shaping their abdomen; they admitted that they were really comfortable using tungku and barut. 


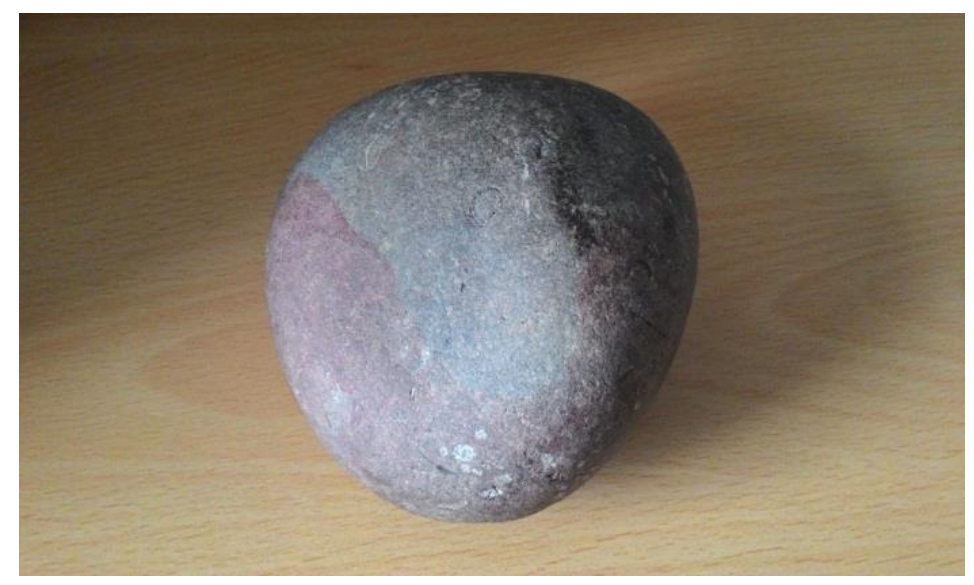

Figure 1: Tungku used by a Malaysian woman in the United Kingdom

In order to heat the body after giving birth, some women replaced tungku with a hot water bottle, which was applied to her body, seeing as it is similar to applying a tungku. For example, Azi, a Malaysian woman, has four children. She gave birth to her first, second, and third children in Malaysia, while the fourth child was born in Great Britain. She always used tungku from the first to third birth, as she felt comfortable using it. However, when she gave birth to her fourth child in Great Britain, she did not have a tungku; therefore, she replaced the tungku with a hot water bottle, which she applied to her body in the same way as a tungku. By means of Azi's experience, I indicated that using tungku is not only a traditional belief but a health belief utilized to maintain a woman's health, as they experience and perceive its function on their own bodies. This demonstrated that prior experiences, which humans have, influence an individual's choices and decisions in the future.

\section{Restricted Foods for The Child-birthing Women}

Heating the mother's body is related to the humoral concept, that the treatments concern the balance restoration. During the post-partum period, when the woman has a subsequent loss of blood and shifts from "hot body fluid" to "cold", she must be treated by prescribing warm substances and keep out of the cold for approximately forty days after the birth of her child. This is known as the confinement period (Zamani, 2001). The Malaysian women in the United Kingdom called it "masa pantang". Therefore, during the postpartum period (a cold state), most women heat their body with hot materials and drink akar kayu ${ }^{1}$, as it helps to restore body heat after blood was lost during delivery, increase blood circulation and prevent illness (Laderman, 1983; Manderson, 1981; Zamani, 2001). In contrast, during the post-partum period, women are strongly recommended not to take "wind foods", as they have "the cold body." The concept of wind (angin) in Malay society linked to the notion of sickness and treatment is believed that wind can bring the dirt and disease which can cause the human body to become ill (Ishak and Nassuruddin, 2014; Laderman, 1991). The concept of 'wind' is also posed in the Chinese concept of illness (Green et al., 2006). It was universal that food selection is to restore the human's body balance and harmony (Green et al., 2006).

The concept of balance restoration was still believed not only by people in Malaysia, but it was also brought to other countries and even Western countries. During the post-partum period, women are

\footnotetext{
${ }^{1}$ Akar kayu means "tonic drinks made from medicinal plants" (Zamani, 2001:31), such as ginger, turmeric, sepang (caesalpiniasappon) and "kederang" (hymenocardiawallichii), etc.
} 
strongly recommended to eat 'hot' foods that can make a cold body warm, such as ginger, turmeric, and black pepper, which are put in their main meals. The suggestion was still practiced by the Malaysian women in the United Kingdom, in particular the ones I interviewed. The women took the culturally recommended foods and medicines and avoided foods that were culturally regarded might be harmful to women and babies. For example, during the post-partum period, the mothers did not eat chicken, eggs, meat, some 'cold' foods, and oily foods. It is believed that eating chicken and eggs might cause the vagina to be itchy, meat is hard to digest, oily foods can cause vaginal tears which take a long time to recover and cold foods might cause women to have 'wind'. As Yy said:

"After birth, I do not eat chicken, egg, and meat, because it can make my vagina itchy. In addition, meat or chicken is difficult to digest. This is advice I received from my family. During the confinement period, it is suggested that the woman consumes hot seasoning, such as ginger, turmeric, and black pepper. It is put on the soup or the food that we eat. If it is hot, then the wind in our bodies can be released, as after giving birth, a woman has a lot of wind in her body."

An, a Chinese-Malay woman, has a different insight of tradition. She did not strictly follow the traditional beliefs, as she received and accepted the insight and suggestion which were contrasted to the humoral concepts. She ate some "cold food" during the confinement period because it was allowed by a health professional, on the other hand, she still consumed the traditional herbal medicines provided by her mother. Certain foods are prohibited during the postpartum period such as fruits and raw, spicy, sour, oily foods (Dennis et al., 2007).

An, informant in this study said "In Malaysia there are a lot of taboos about what a mother should not eat during the post-partum period, such as 'wind food' and 'cold food' (makanan yang sejuk) like orange and other certain fruits. But, when I asked the midwife here, there are not any taboo foods for the post-partum mother and she told me that the mother is allowed to eat everything, I followed what the midwife suggested. For example, after giving birth, the midwife gave me fish and chips, ice cream and oranges, which the post-partum mother in Malaysia is not allowed to consume it. But, I ate all these foods. When my mother saw these foods, she prohibited me, although I said "It is allowed and provided in hospital, and I asked the midwife." Then, she accepted my argument. As a Chinese-Malay woman, my mother cooked herbal Chinese medicine for me during the post-partum period. I do not actually know what it contains, but I know it is made from root-wood (akar kayu). She brought the medicine from Malaysia, but I think it is also provided in the Chinese market. She cooked akar kayu together with chicken soup. My mother cooked it every day and said it is good for enhancing the blood after birth."

Social and community influences are determinants influencing a human's health as possible as cultural and environmental condition (Davies et al., 2006). Social-cognitive factors, which women encounter, have influenced and shaped the food options of pregnant and post-parturient women (Padmanabhan, Summerbell and Heslehurst, 2015:2). Therefore, each group of people has their own ways and beliefs pertaining to restricted foods and medicines, which should be taken by women during pregnancy and the postpartum period. People who live nearby have an essential role in helping women maintain and treat their bodies during pregnancy and the postpartum period, as they offer experience and knowledge. For example, Asy, who unfortunately experienced a miscarriage during her first pregnancy, told me that the other Malaysian women told her what foods she should and should not eat after a miscarriage to make a full recovery. Additionally, they also taught her how to 
use tungku, whereas, in the past, Asy did not know these traditional beliefs and practices. In addition, after she had the miscarriage, the other Malaysian women took their turn to cook for her for roughly 14 days. Similarly, Yaya also said that if a Malaysian woman gives birth, the other Malaysian women will cook for her for approximately 14 days. This revealed that the treatment and care of women's bodies after postpartum is usually supported by the society they live in.

Social support is given to the new mother after birth shifts temporarily the mother's role from who is usually caregiver to the all family members to who needs attention more (Dennis et al., 2007). In my opinion, the mother getting more careful during postpartum is not to have fully cured, but to force the mother not to lose their energy to care for the whole family members when she had passed the postpartum period.

\section{Discussion}

The WHO (2013) stated that the days after birth, known as the postnatal period is a critical phase for mothers and newborns, as most maternal and infant deaths occur during this time. Therefore, (WHO, 2015) strongly recommended that health professional in all countries should provide four visits of postnatal care for mothers and newborn: 1) first day (24 hours after birth), 2) third day (48-72 hours after birth), 3) between days 7-14, and 4) six weeks after birth. The home visits have to be undertaken by midwives or skilled and well-trained health workers. However, the midwives often deny traditional treatments as they studied midwifery based on the Western perspectives. As a result, midwives often deny traditional treatment. The people who skeptical of non-Western medicine assume it is not scientific medicine as it is only the common medicine made within the family (Green et al., 2006). On the other hand, the women, either from Chinese or other ethnicities, use the traditional treatment as a supplement rather than substitute the biomedical medicines (Green et al., 2006).

When the mother finds the denial views from the midwife, she repeatedly practices the traditional treatments furtively. Mr, a mother's whom I interviewed, said that she will take off the barut when the midwife or health visitor came to her house for visiting, and she admitted that the midwife or health visitor did not even know the heating tradition. The woman concealed the practice because she was afraid that the midwife would prohibit the tradition. Therefore, in my opinion, the midwife should learn the traditional treatments and moreover, there is a lot of immigrant coming from all over the world with different cultures to the United Kingdom. Understanding the traditional practice during the postpartum period can bring the perinatal services appropriate with cultural competence (Dennis et al., 2007).

The traditional treatment is considered oftentimes that it is practiced only by the people who live far away from the health facility, have inadequate health knowledge, and have low education. However, we can see that informants in this study were well educated and even some of them were postgraduate students, but they still practiced the traditional treatment. This confirmed that education and occupation are not related to the women's choice of treatment, on the condition that they perceived that the treatment has a positive effect on their bodies. Laderman (1983) also argued although the use of a roasting bed and tungku remains contentious among people, these traditions are still practiced by many people in Southeast Asia, and even by people with higher education. Furthermore, (Green et al., 2006) study revealed that the Chinese migrant who migrated to England since she was a child, spoke fluent English, and had private medical insurance, came to the Chinese spiritualists to have treatment 
as she had not got better when she was medicalized by the western practitioner. In conclusion, well education and fluent language are not the underpinned factors of the people practicing and believing the traditional values. Although the migrants could be able speaking a fluent host language to communicate to the medical practitioners, it can deter misunderstanding and communication problems because of the different ideas and concepts of symptoms and illness (Green et al., 2006).

National boundaries had not obstructed people from utilizing their own cultural values in other countries, as they perceived and experienced the benefits of cultural practices on their own bodies. More interestingly, the traditional practices were done in modern countries, where medicalization or high technology was born. Although the use of Western biomedicine has been dominant in the Western community, the alternative healing medicine now has been also accepted and used widely by most populations (Green et al., 2006). Western and Chinese medical knowledge are clearly different. Where in Western knowledge, health and illness are related to the anatomic parts of the human body, in the Chinese perspective of health, illness and body are a holistic approach of the energy flow of the human body and related to the idea of balance (Green et al., 2006).

Western postpartum care for both new mother and infant focuses fundamentally on the mother's and baby's immediate physical health by using medical technology intervention (Posmontier \& Horowitz, 2004). In non-western cultures, the family members such as mother, female relatives, mother-in-law, spouse, and community usually take care of the new mother and infant, such as cooking and household chores as well as information for the woman how to care for herself and baby (Dennis $e t$ al., 2007). The traditional practices for postpartum mothers in some ethnicities intend to avoid mental illness and infertility in the future, as a result of incomplete traditional practices (Dennis et al., 2007). In many cultures, the new mother should eat certain foods to restore health, and avoid prohibited foods which belief can cause the illness immediately or later years (Dennis et al., 2007).

The place of origin played important roles in the women's pathways and decision to use the treatment for their health (Green et al., 2006). People frequently move to another country where the cultural values and traditional norms are different from their country of birth. This the big challenge for women to adhere to cultural norms and moreover when they have limited access to the people out of their community (Davies et al., 2006). For example, Malaysian women prefer to choose the female medical professional as it is related to their cultural values, whereas in the United Kingdom the medical professional for everyone has been determined. Migrant-friendly health services have to be able to respond to the particular problems related to the norms and values, in particular women, who frequently face difficult access to the health care service where they should be seen by the men whereas in their norms it was prohibited (Davies et al., 2006).

Language barriers may cause communication problems because frequently there is a different concept of illness between the patients and medical professionals as they are coming from different cultures and values. As a result, the migrants prefer to seek help from traditional healers (Davies et al., 2006) or practice the traditional healing from their cultures. Health policy implicitly regards the migrants might adopt the health perspectives and beliefs of the destination country (Davies et al., 2006). Different health beliefs and knowledge can obstruct access to health care services. Therefore, incorporating the medical dualism in the health systems can enhance the cultural appropriateness in the health care systems, and migrants could contribute to making the health system appropriate and acceptable to migrants (Davies et al., 2006). 
The sophisticated technology and modern health services have not influenced people's choice of treatment when they have felt comfortable using traditional practices on their bodies. Honestly speaking, the reason why people use cultural traditions is not that they are not satisfied with the health services, but also because they perceived and experienced the value of cultural tradition on their bodies. Therefore, I have indicated here that policymakers in the developed countries, where there are a lot of migrants coming from different cultures, have to consider the traditional treatments in the health systems.

\section{Conclusion}

Nowadays, certain societies still believe in and use traditional treatments during pregnancy, birth, and postpartum periods, and even regard these methods and treatments as the best way to assist women to remain healthy and well during pregnancy and after birth. In addition, people who live around women, in a particular family or a colleague who has the same ethnicity, culture, religion, or nationality, also have an influence on how women perceive and deal with pregnancy and birth. Although the women perceived and experienced good quality health services during pregnancy, giving birth, and the postpartum period, they still also carried out the cultural traditions of their families and societies, in particular, tungku and barut for a mother during the postpartum period. Surprisingly, they brought these tools from their home countries. In addition, they also still obey the cultural values concerning post-parturient women, for example, what they should and should not consume during the postpartum period.

There are two principal reasons why women still undertake these traditions. Firstly, those who experienced childbirth in their hometown, where they learned about the cultural traditions, felt and perceived that the tradition is good for their health since they felt that their bodies were healthier after using traditional practices, such as tungku and barut. This revealed that bodily experience influenced people's decisions with regards to the treatment of their bodies. Secondly, for those who have not experienced childbirth in their hometown, frequently it is the woman's first delivery, learned about the tradition from people who lived in the community around her, such as family or friends from the same country. This proves how society influences how people treat their bodies. However, it needs further study to reveal the effectiveness and efficacy of using tungku on women's body, moreover the study has a lot of limitation.

\section{Statement Regarding Informed Consent}

All informants involved in this study had been asked for their agreements, which were signed in the letter of agreements.

\section{Statement Regarding Ethical Approval}

The ethical clearance of the study has been approved by the Department of Anthropology, Durham University United Kingdom. 


\section{References}

Bernard, R. (2006). Research Methods in Anthropology; Qualitative and Quantitative Approaches (Fourth). Altamira Press.

Browner, C. H., \& Sargent, C. F. (2011). Toward Global Anthropological Studies of Reproduction. In Reproduction, Globalization and The State; New Theoritical and Ethnographic Perspecttives (pp. 1-18). Duke University Press.

Davies, A., Basten, A., \& Frattini, C. (2006). Migration: A social determinant of migrants' health. IOM.

Dennis, C.-L., Fung, K., Grigoriadis, S., Robinson, G. E., Romans, S., \& Ross, L. (2007). Traditional Postpartum Practices and Rituals: A Qualitative Systematic Review. Women's Health, 3(4), 487-502. https://doi.org/10.2217/17455057.3.4.487

Dundes, L. (2003). The Manner Born: Birth Rites in Cross-cultural Perspective. Rowman Altamira.

Green, G., Bradby, H., Chan, A., \& Lee, M. (2006). "We are not completely Westernised": Dual medical systems and pathways to health care among Chinese migrant women in England. Social Science \& Medicine (1982), 62(6), 1498-1509. https://doi.org/10.1016/j.socscimed.2005.08.014

Henley-Einion, A. (2003). The Medicalisation of Childbirth. In The Social Context of Birth. Radcliffe Medical Press Ltd.

Ishak, S., \& Nassuruddin, M. G. (2014). Traditional Malay Healing Practices: Expressions of Cultural and Local Knowledge. Procedia - Social and Behavioral Sciences, 140, 291-294. https://doi.org/10.1016/j.sbspro.2014.04.422

Jordan, B. (1992). Birth in Four Cultures: A Crosscultural Investigation of Childbirth in Yucatan, Holland, Sweden, and the United States, Fourth Edition. Waveland Press.

Laderman, C. (1983). Wives and Midwives: Childbirth and Nutrition in Rural Malaysia. University of California Press.

Laderman, C. (1991). Taming the Wind of Desire: Psychology, Medicine, and Aesthetics in Malay Shamanistic Performance. University of California Press.

Lupton, D. (2007). Medicine as Culture (Second). Sage Publications.

Manderson, L. (1981). Roasting, smoking and dieting in response to birth: Malay confinement in cross-cultural perspective. Social Science \& Medicine. Part B: Medical Anthropology, 15(4), 509-520. https://doi.org/10.1016/0160-7987(81)90025-9

Padmanabhan, U., Summerbell, C. D., \& Heslehurst, N. (2015). A qualitative study exploring pregnant women's weight-related attitudes and beliefs in UK: The BLOOM study. BMC Pregnancy and Childbirth, 15, 99. https://doi.org/10.1186/s12884-015-0522-3

Posmontier, B., \& Horowitz, J. A. (2004). Postpartum practices and depression prevalences: Technocentric and ethnokinship cultural perspectives. Journal of Transcultural Nursing: Official Journal of the Transcultural Nursing Society, 15(1), 34-43. https://doi.org/10.1177/1043659603260032

WHO. (2013). Postnatal Care of the Mother and Newborn. http://www.who.int/maternal_child_adolescent/publications/WHO-MCA-PNC-2014-Briefer_A4.pdf

WHO. (2015). WHO | WHO recommendations on postnatal care of the mother and newborn. WHO. http://www.who.int/maternal_child_adolescent/documents/postnatal-care-recommendations/en/

Zamani, A. (2001). Traditional Practices in Postnatal Care: The Malay Community in Malaysia. Trinity Student Medical Journal, 2, 30-31. 